\title{
CMS Hadron Forward Calorimeter Phase I Upgrade Status
}

\author{
Burak Bilki*i \\ University of Iowa, Iowa City, IA 52242 USA \\ Argonne National Laboratory, Argonne, IL 60439 USA \\ E-mail: burak-bilki@uiowa.edu \\ Yasar Onel \\ University of Iowa, Iowa City, IA 52242 USA \\ E-mail: yasar-onel@uiowa.edu
}

\begin{abstract}
The Hadron Forward Calorimeter of CMS is going through a complete Phase I upgrade. The current photomultiplier tubes (PMTs) are being replaced with thinner window, higher quantum efficiency, four-anode photomultiplier tubes. The new PMTs will provide better light detection performance, a significantly reduced background and unique handles to recover the signal in the presence of background. This report will describe the nature of the essential upgrade elements with supporting beam test results and the status of the upgrade progression.
\end{abstract}

Technology and Instrumentation in Particle Physics 2014,

2-6 June, 2014

Amsterdam, the Netherlands

\footnotetext{
*Speaker.

†n behalf of the CMS Collaboration
} 


\section{Introduction}

The Compact Muon Solenoid (CMS) [1] is a general-purpose detector designed to run at the highest luminosity provided by the CERN Large Hadron Collider (LHC). Coverage between pseudorapidities $(\eta)$ of 3 and 5 is provided by the steel/quartz fiber Hadron Forward (HF) calorimeter which is located at $11.2 \mathrm{~m}$ from the interaction point. The signal originates from Čerenkov light emitted in the quartz fibers, which is then channeled by the fibers to photomultipliers. Machining 1 $\mathrm{mm}$ square grooves into steel plates, which are then diffusion welded together, creates the absorber structure that is $1.65 \mathrm{~m}$ long. The diameter of the quartz fibers is $0.6 \mathrm{~mm}$ and they are placed $5 \mathrm{~mm}$ apart in a square grid. The quartz fibers, which run parallel to the beam line, have two different lengths $(1.43 \mathrm{~m}$ and $1.65 \mathrm{~m})$, which are inserted into grooves, creating two effective longitudinal samplings. The so-called "short fibers" start $22 \mathrm{~cm}$ inside the absorber, hence are mostly sensitive to hadron interactions. There are 13 towers in $\eta$, and the $\phi$ segmentation of all towers is $10^{\circ}$, except for the highest $\eta$. This leads to 900 towers and 1800 channels in the two HF calorimeter modules [2]. Details of the HF calorimeter design, together with test beam results and calibration methods, can be found in [3].

\section{Description of the Upgrade and the Key Components}

The photomultiplier tubes (PMTs) of the CMS HF calorimeter generate a large, fake signal when the PMT window is traversed by a relativistic charged particle due to Čerenkov light production at the PMT window. These PMTs have circular windows with $2.54 \mathrm{~cm}$ diameter and $2 \mathrm{~mm}$ thickness at the center that gets thicker towards the rim (Hamamatsu R7525 [4]). This alreadyknown problem was observed in the 2010 and 2011 CMS data to degrade data quality and to constitute a potential to interfere with rare physics events. An upgrade plan was therefore formulated for CMS HF. In this framework, several types of PMTs were tested and the four-anode R7600U200-M4 by Hamamatsu [4] was selected as the replacement PMT for the upgrade [5]. The upgrade PMT has a square window of size $1.8 \mathrm{~cm} \times 1.8 \mathrm{~cm}$ and thickness less than $1 \mathrm{~mm}$ indicating a significant reduction in the amount of glass seen by the traversing relativistic particles. The new PMT not only reduces the intrinsic level of background, but it also enables tagging of background events and recovering the underlying signal event (if any) by using the multi-anode features.

A readout box (RBX) prototype was built to enable the tests of different readout options for the new four anode PMTs. The new readout boards provide the flexibility to switch between fourchannel, two-channel and single-channel readout of the four anode PMTs where the four-channel readout option enables the full multi-anode functionality. Both the internal and external cabling of the RBX were also specific designs and selections, therefore form an integral part of the prototype.

The prototype RBX was tested in the CERN H2 beam line [6] in Summer - Fall 2011 with electron and muon beams to mimic calorimeter and background responses respectively.

HF front-end and back-end electronics will be upgraded in order to support multi-channel readout of the R7600 PMT. Testing has been completed and commissioning is ongoing, with a goal to install before Long Shutdown 2, which is projected to be in 2018 - 2019.

The front-end electronics, named QIE10 (Charge Integrator and Encoder) [7], are radiation tolerant (up to $100 \mathrm{~Gy}$ ) and include the addition of a TDC circuit. The TDC has a rising edge 
resolution of 390 to $780 \mathrm{ps}$ ( 5 or 6 bits). This next generation QIE card has deadtimeless integration and digitization of charge in $25 \mathrm{~ns}$ buckets, large dynamic range ( $3 \mathrm{fC}$ to $330 \mathrm{pC}$ ) stored in 17 bits, and has digitization error less than the calorimeter resolution (2-3\%). This increased data will require a new pipeline, and the SLHC beam conditions will require a radiation hard solution. The GigaBit Transceiver (GBTx) technology will serialize data from the QIE10 cards and transmit the data to the back-end electronics in the counting room. 288 new GBT data-link fibers will allow for the addition of the TDC bits, including the presence of multiple rising or falling edges. Upgraded HF back-end electronics will replace the current VME based system and will be installed into an industrial-standard microTCA crate. This is the first step of the Phase I electronics upgrade for HF.

\section{Upgrade Status}

Nearly 1800 upgrade PMTs (R7600s) were tested at the University of Iowa. The system involved: 3 dark boxes, a VME and CAMAC data acquisition system, an XY scanner for surface linearity testing, 3 picoammeters, 3 digital oscilloscopes, UV and visible light power meters, UV and Blue LED light sources, 2 nitrogen lasers, DC tungsten light bulbs, optical tables for mounting optical elements in the dark boxes, picosecond LED pulsers and 1 double pulse generator.

Reusing as much existing readout box infrastructure as possible was a major design goal of the project, while still meeting all criteria for proper PMT operation. Another consideration was the flexibility to change the number of channels read out per PMT. This capability became part of the baseboard design. These design considerations were prototyped and tested in CMS in the fall of 2010 during collisions with great success.

A slice-test readout box was designed and prototyped to directly compare HF PMT and R7600 readout in a real collision environment. The slice test mixed $20 \mathrm{R} 7525 \mathrm{~s}$ with 8 4-anode R7600 PMTs. This RBX was placed in HF at iPhi 67 and covered towers iEta 29 through iEta 32 in HF+. The R7600 placed at coordinate (iPhi 67, iEta 29) was blacked out so only Čerenkov light generated by MIP interactions would be recorded. Analysis of the collision data was consistent with test beam data, namely the mean energy of the MIP event reduced from $120 \mathrm{GeV}$ to $35 \mathrm{GeV}$, and the rate was significantly lower.

New shields for the R7600 PMTs had to be manufactured, and the success of the pre-production prototype demonstrated that the design considerations are well understood.

The upgrade readout box was tested at the CERN $\mathrm{H} 2$ test beam facility [8]. The operation was very successful, with no abnormal signals and no significant issues. The results show that the performance characteristics are well understood, and the R7600 PMTs have a uniform response under different readout conditions (1,2, or 4 anode readout). A major success was the negligible response of MIPs with the tagging algorithm. The efficiencies of the algorithms were quantified with $98 \%$ for 4 -ch readout and $90 \%$ for 2 -ch readout. The 4 -channel readout option has more controlling power on background elimination and is an unprecedented readout segmentation in an operational calorimeter.

By the time this note is written, the upgrade readout boxes are being assembled and tested at CERN. Following the installation and commissioning, the upgrade HF will be operational in 2015. 


\section{Summary}

The HF Phase I upgrade is underway with the construction of the upgrade readout boxes with complete upgrade components and readout options as of the day this note is written.

The multi-anode upgrade PMTs do not only reduce the rate of the background as a result of their physical specifications, but also enable the implementation of signal recovery algorithms pertaining to the multi-anode features. The design of the upgrade readout and adapter boards enables simple operation to switch between 1,2 and 4-channel readout options. The new cabling significantly improves the space issues.

The front- and back-end electronics are developed to enable precision TDC for detailed signal shape information. The electronics is also associated with fast data links and is radiation hard.

All the upgrade components are selected to provide the Hadron Forward calorimeters operations in the upgrade era with the desired performance characteristics. The components are extensively tested and the upgrade concept is validated with several beam tests. Progression of the upgrade is on schedule towards commissioning and operation planned in 2015.

\section{References}

[1] S. Chatrchyan et al. (CMS collabration), The CMS experiment at the CERN LHC, JINST 3 S08004, 2008.

[2] G. L. Bayatian et al. (CMS collabration), CMS physics: Technical design report, CERN/LHCC-2006-001, 2006.

[3] S. Abdullin et al. (CMS HCAL collabration), Design, performance, and calibration of CMS forward calorimeter wedges, Eur. Phys. J. C 53 139, 2008.

[4] http://www.hamamatsu.com/

[5] S. Chatrchyan et al. (CMS HCAL collaboration), Study of various photomultiplier tubes with muon beams and Cerenkov light produced in electron showers, JINST 5 P06002, 2010.

[6] http://nahandbook.web.cern.ch/nahandbook/default/

[7] T. Zimmerman and M. Sarraj, A second generation charge integrator and encoder ASIC, IEEE Trans. Nucl. Sci. 43 1683, 1996; T. Zimmerman and J.R. Hoff, The design of a charge-integrating modified floating-point ADC chip, IEEE J. Solid State Circ. 39 895, 2004.

[8] S. Chatrchyan et al. (CMS HCAL collaboration), Test of CMS hadron forward calorimeter upgrade readout box prototype, JINST 7 P10015, 2012. 